\title{
Estereotipos y Roles de Género en la Evaluación Laboral y Personal de Hombres y Mujeres en Cargos de Dirección
}

\section{Gender Stereotypes and Roles in the Labor and Personal Evaluation of Men and Women in Management Positions}

\author{
Lorena Godoy \\ Universidad Diego Portales
}

\author{
Antonio Mladinic \\ Pontificia Universidad Católica de Chile
}

\begin{abstract}
En base a la teoría de la congruencia de roles (Eagly, 1987), este estudio analizó cómo estereotipos y roles de género afectaban la evaluación que recibía un hombre y una mujer gerente. El estudio fue cuasi experimental, transversal e inter sujeto. Participaron 242 ejecutivos, quienes debían leer la descripción de un gerente hombre o mujer y evaluarle en varios dominios laborales y personales. Se realizaron análisis univariados, multivariados y de mediciones repetidas de la varianza, considerando sexo del evaluado y del participante y ámbito (laboral y personal). No se encontraron diferencias en las evaluaciones con respecto al sexo de los participantes o de los evaluados. Sí se encontró un efecto principal del ámbito, recibiendo gerentes hombres y mujeres una evaluación mayor en el entorno laboral que en el personal.
\end{abstract}

Palabras clave: estereotipos y roles de género, trabajo, evaluación de desempeño, mujeres.

\begin{abstract}
Based on the congruence role theory (Eagly, 1987), this study analyzed how gender stereotypes and gender role expectations influenced the evaluations of either a female or a male executive officer. The study was quasi-experimental and transversal, with a mixed design. Two hundred and forty two managers were asked to read the description of an executive officer and evaluate the target on various domains in both work and personal settings. One-way, factorial, and repeated measures analysis of variance were performed, considering target's and participant's sex and setting (work or intimate setting). The results showed that neither the participant's nor the target's sex influenced evaluations. However, a main effect was found for setting, such that both men and women were better evaluated in the working setting than in the personal setting.
\end{abstract}

Keywords: gender stereotypes, gender roles, work, performance evaluation, women.

\section{Introducción}

\section{La Creciente Presencia de Mujeres en el Mercado Laboral}

La feminización de la fuerza laboral constituye uno de los fenómenos más relevantes ocurridos en los mercados laborales mundiales en las últimas décadas (Organización Internacional del Trabajo [OIT], 1997, 2007; Valenzuela \& Reinecke, 2000), lo que se evidencia en distintos países -incluido Chile- en un aumento del número de mujeres que se integran al mercado de trabajo, un crecimiento más rápido de sus tasas de ocupación en relación a las de los hombres, un aumento de sus años activos y un aumento de empleos técnicos y profesionales ocupados por ellas. Esto se relaciona con una serie defactores: la disminución de las tasas de fecundidad, un mayor acceso a la educación, mayor necesidad de los hogares de contar con mayores ingresos, crisis económicas y patrones culturales que favorecen el ejercicio por parte de las mujeres de roles tradicionalmente asociados a los hombres (Abramo \& Valenzuela, 2006).

Lorena Godoy, Escuela de Psicología, Universidad Diego Portales, Santiago, Chile; Centro de Estudios de la Mujer, Santiago, Chile.

Antonio Mladinic, Escuela de Psicología, Pontificia Universidad Católica de Chile, Santiago, Chile.

La correspondencia relativa a este artículo debe ser dirigida a Antonio Mladinic Alonso, Escuela de Psicología, Pontificia Universidad Católica de Chile, Avda. Vicuña Mackenna 4860, Macul, Santiago, Chile. E-mail: amladinic@uc. cl,con copia a lgodoy@cem.cl, lgodoyc@uc.cl

El estudio presentado corresponde a la tesis de Magíster en Psicología Social Comunitaria de la Pontificia Universidad Católica de Chile de la primera autora. Para su realización contó con una beca de la Fundación Volcán Calbuco. 
Diversos estudios han demostrado que, dependiendo de las condiciones en las que se da esta inserción laboral, el empleo femenino ha contribuido a que las mujeres aumenten sus grados de independencia personal, su autonomía económica y sus posibilidades de realización personal, todo lo cual ha tensionado roles tradicionalmente asignados a hombres y mujeres en el ámbito de la producción y la reproducción, respectivamente (Corporación Humanas, 2007; Díaz, Godoy \& Stecher, 2005; Godoy, Stecher \& Díaz, 2007; Grupo Iniciativa Mujeres, 1999; Guzmán, Mauro \& Araujo, 1999; Rivera \& Guajardo, 1996; Servicio Nacional de la Mujer [SERNAM], 2001; Sharim \& Silva, 1998).

Junto a lo anterior, se asiste a un reconocimiento de la conveniencia de la incorporación de ciertas características asociadas a las mujeres para el funcionamiento de las organizaciones, destacándose el tema del liderazgo ${ }^{1}$. Se ha señalado que hoy las organizaciones necesitan transformar sus estructuras altamente jerarquizadas por otras horizontales, flexibles y multiculturales, y adoptar estilos de liderazgos transformacionales (Bass \& Avolio, 1994; Bass, Avolio, Jung \& Berson, 2003; Eagly \& Carli, 2007; Sarrió, Ramos \& Candela, 2004). Investigaciones en psicología social califican este estilo de liderazgo como más femenino, caracterizado como más conectivo e interactivo, dirigido a generar redes, nutrir relaciones y dar poder a las personas. Tres metaanálisis sobre investigaciones psicosociales acerca de liderazgo y género realizadas hasta la década del noventa concluyen que a la hora de dirigir, las mujeres son más "democráticas" que los hombres y que ellos son más "autocráticos" (Eagly \& Karau, 1991; Eagly, Karau \& Makhijani, 1995; Eagly, Makhijani \& Klonsky, 1992). Más recientemente, otros estudios confirman que, si bien hombres y mujeres asumen conductas

\footnotetext{
1 En Chile, Hola y Todaro (1992) advertían que desde mediados de los ochenta la literatura empresarial visualizaba ciertas características atribuidas tradicionalmente a las mujeres (intuición o emocionalidad) como virtudes útiles especialmente para cargos directivos. Algunos autores han denominado estas habilidades como destrezas blandas, que convertirían a las mujeres en especialistas emocionales de las relaciones (Reygadas, 1998) y podrían propiciar formas más horizontales de ejercicio del poder.
}

de diferentes tipos de liderazgos, las mujeres líderes tienden a adoptar un estilo más transformacional, el cual sería más efectivo para los actuales contextos organizacionales (Eagly \& Carli, 2004, 2007; GarcíaRetamero \& López-Zafra, 2006).

\section{Persistencia de Procesos de Segregación}

Sin embargo, estas mayores tasas de participación de las mujeres en la fuerza laboral "por sí solas no implican necesariamente que los mercados de trabajo estén evolucionando en forma positiva para las mujeres" (OIT, 2007, p. 3). Se advierte la persistencia de procesos de discriminación que imponen una serie de obstáculos al desarrollo de las trayectorias laborales de las mujeres en igualdad de condiciones que los hombres. Entre los más importantes se encuentran la diferencia de remuneraciones entre hombres y mujeres, la segregación horizontal (los empleos femeninos se concentran en un número reducido de sectores de actividad y de profesiones) y la segregación vertical (concentración de los empleos femeninos en las categorías que se encuentran en la parte baja de la jerarquía) (Maruani, 1993). Así, aunque las brechas salariales han disminuido en las últimas décadas, ellas aún existen: en Chile en el año 2006 el ingreso de las mujeres representaba el $76,6 \%$ del de los hombres y, entre mujeres con mayor educación, el porcentaje era de 61,4\% (Chile, Ministerio de Planificación, 2006). En el año 2002 el 87\% de las mujeres se concentraba en las ramas de servicios (comercio, enseñanza y servicios domésticos). $\mathrm{Y}$, si bien en diversos países se observa un aumento de las mujeres en cargos gerenciales, ellas siguen siendo una minoría. En Chile, en las directivas de organizaciones empresariales ellas aumentaron de 4 a 10 entre $1991 \mathrm{y}$ 2001 (SERNAM, 2005), entre los miembros del poder ejecutivo, legislativo y personal directivo de la administración pública y de empresas representaban, según el Censo de 2002 , un $32,7 \%$ y, entre las 40 empresas que integran el IPSA de la Bolsa de Comercio de Santiago, solo un $2,11 \%$ tienen mujeres en sus directorios, cifra que aumenta a $4 \%$ al considerar el total de empresas de sociedades anónimas -abiertas y cerradas- (Centro de Estudios Empresariales de la Mujer, 2008). 


\section{La Escasa Presencia de Mujeres en Cargos de Dirección}

La escasa presencia de mujeres en los niveles jerárquicos más altos de las organizaciones es el fenómeno en el cual se centró el estudio. Se trata de una situación que, por una parte, demuestra las nuevas oportunidades que les ofrece el mercado a las mujeres y los mayores niveles de escolaridad que han alcanzado y, por otro lado, refleja las dificultades y problemas que encuentran para ascender a niveles altos de la jerarquía y para desempeñarse en dichos niveles, donde se agudizan algunos de los procesos de segregación laboral por género que afectan al conjunto de las mujeres.

Diversos argumentos han surgido para explicar esta escasa presencia de mujeres. Se ha señalado que ellas carecen de la experiencia laboral necesaria (teoría del déficit, Heilman, 2001) o que existiría una falta de interés o motivación por este tipo de cargos, pues tendrían una concepción "altruistaafectiva" del trabajo (Hola \& Todaro, 1992; Pratto, Stallworth, Sidanius \& Siers, 1997, citado en Myers, 2005). El hecho que las responsabilidades familiares sigan recayendo predominantemente en las mujeres ha sido señalado por aquellas que se desempeñan en este tipo de cargos como uno de los obstáculos más importantes para acceder a estas posiciones. Eagly y Carli (2007) afirman que las demandas de la vida familiar hacen que las mujeres interrumpan más sus carreras y trabajen más en jornadas parciales, lo que se traduce en menos años de experiencia y menos horas de empleo por año, en comparación con los hombres. Esto lentifica el progreso de sus carreras, reduce sus ingresos y limita su tiempo para construir redes profesionales que pueden resultar cruciales en el avance de sus trayectorias laborales. Es decir, más que desinterés por asumir estos cargos, las mujeres enfrentarían mayores limitaciones.

Aquellas que se desempeñan en este tipo de cargos también advierten la presencia de ciertas ideas respecto de la capacidad de mujeres y hombres para desempeñarlos, que les obstaculizan acceder a ellos. Una investigación realizada por Catalyst (2007), que consultó a mujeres directivas en empre- sas en Estados Unidos y en diversos países europeos, plantea que las mujeres en posiciones de liderazgo se enfrentaban a diversos dilemas, entre ellos, tener que cumplir con altos estándares y recibir bajas recompensas. El percibir a las mujeres como líderes atípicas las obligaba a probar que podían dirigir, incluso antes de tener la oportunidad de hacerlo. Y por ello debían gastar tiempo y energía adicional probando sus capacidades, lo que redundaba en una mayor carga de trabajo, en comparación con sus compañeros hombres, para probar el mismo nivel de competencia. En América Latina, en un estudio que consultó a 120 mujeres directivas de empresas de siete países (Maxfield, 2005), una amplia mayoría aseguró que su éxito se debía a que trabajaba más que sus colegas hombres para obtener el mismo reconocimiento: "la percepción de las mujeres es que sus colegas, subordinados y superiores, les someten a diversas y ocultas formas de evaluación o pruebas de desempeño y que implícitamente se les juzga con un estándar superior a aquel con el que se juzgaría a los hombres" (p. 17). En un estudio realizado en Chile, en que se analizaron las razones del desigual estatus de mujeres ingenieras en el ámbito de la investigación en ciencia y tecnología, las entrevistadas indicaron que algunas de las dificultades que enfrentaban en un campo profesional tradicionalmente dominado por los hombres, como es la ingeniería, se agudizaban cuando estaban a cargo de equipos de investigación. En esas situaciones, la necesidad que sentían de demostrar constantemente sus capacidades se acentuaba, debiendo hacer grandes esfuerzos para ser respetadas y tratadas como jefas, especialmente cuando tenían hombres bajo su dirección. Además de demostrar sus competencias profesionales, debían asumir ciertos rasgos, tales como carácter fuerte, agresividad y autoridad, asociados tradicionalmente a los hombres, para ser aceptadas como líderes. Una entrevistada señalaba al respecto que ellas tenían que "endurecerse" para ser reconocidas como líderes (Yáñez \& Godoy, 2008).

Eagly y Carli (2007) han planteado la figura del laberinto para graficar estas dificultades que enfrentarían las mujeres. Esta figura transmite la idea que, más que una 
obstrucción transparente, invisible y sutil, y ubicada en un nivel específico de la organización, cercano a las más altas posiciones jerárquicas, como lo sugería la figura del techo de cristal (OIT, 1997), los obstáculos que enfrentan las mujeres son múltiples y en diferentes momentos de sus trayectorias laborales, y que no siempre son invisibles para ellas: prejuicios sobre los intereses que guían sus trayectorias laborales y sobre su presencia en cargos de dirección, particulares resistencias al liderazgo femenino y aspectos que desafían el balance entre trabajo y responsabilidades familiares.

Lo que se desprende de estos distintos estudios es que muchas de las dificultades más importantes que deben enfrentar las mujeres que quieren acceder a cargos de dirección se relacionan con asociaciones conscientes e inconscientes, ampliamente compartidas, que predominantemente relacionan rasgos y conductas estereotípicamente masculinas con roles de liderazgo. Al mismo tiempo, se percibe una incongruencia entre las cualidades y conductas predominantemente atribuidas a las mujeres y las cualidades necesarias para desempeñar cargos de dirección o liderazgo.

\section{La Teoría de la Congruencia del Rol}

Estas asociaciones que pueden dar origen a procesos de discriminación hacia mujeres en el camino por ocupar cargos directivos son explicadas en el presente estudio desde la teoría de la congruencia de rol planteada por Eagly (1987). La autora indica que muchas de estas asociaciones expresan expectativas compartidas sobre la conducta de las personas en base a su sexo socialmente identificado, es decir, expresan roles de género. Dichos roles contienen normas descriptivas, expectativas consensuadas respecto de las conductas típicas de hombres y mujeres y normas prescriptivas, conductas deseables o admiradas de cada sexo probables de elicitar aprobación de los demás y proveer sentimientos de orgullo o vergüenza (Eagly, Wood \& JohannesenSchmidt, 2004; Heilman, Wallen, Fuchs \& Tamkins, 2004). Los roles de género se basan en las diferentes posiciones que ocupan hombres y mujeres en la división sexual del trabajo, y las personas infieren que dichos roles reflejan atributos personales, propiedades inherentes o disposiciones internas (sesgo de correspondencia, Eagly et al., 2004). Así, el hecho que las mujeres desarrollen roles familiares, conductas (roles) principalmente relacionadas con el cuidado y bienestar de otros (cuidado infantil y labores domésticas), hace que las personas infieran que existe una relación con sus rasgos o disposiciones internas, las que reflejan precisamente el interés por el bienestar del otro: sensibilidad interpersonal, calidez, amabilidad, agradabilidad y empatía. Y en el caso de los hombres, el que desarrollen conductas (roles) dominantes, de mayor estatus y poder, produce expectativas de que ellos posean rasgos tales como asertividad, autonomía, actividad e independencia. Siguiendo la denominación planteada originalmente por Bakan (1966, citado en Eagly, 1987), Eagly denomina a los rasgos atribuidos preferentemente a las mujeres como comunales y a los atribuidos preferentemente a los hombres como agénticos. Sin embargo, es importante señalar que ninguna de estas atribuciones de rasgos estereotípicos es extrema: los sexos se piensan como diferentes pero no extremadamente diferentes, pudiendo hombres y mujeres presentar, en distintos niveles, rasgos agénticos y comunales ${ }^{2}$.

Específicamente respecto del rol de liderazgo, diversas investigaciones indican que mujeres y hombres perciben que las características asociadas al éxito en cargos directivos se corresponden fuertemente con las características agénticas, generalmente adscritas a los hombres. Este fenómeno, denominado por Schein (2001, p. 676) como "pensar en un gerente es pensar en un hombre" (think manager-think male), constituiría una de las barreras psicológicas más importantes que enfrentan las mujeres para acceder a cargos de dirección.

\footnotetext{
2 Rudman y Glick (2001) indican que el estereotipo comunal es típicamente atribuido a un grupo de bajo estatus en sistemas estables de inequidad, como son las relaciones de género. Y está asociado con la conducta deferente que los dominantes demandan de los subordinados; por lo tanto, tiende a ser altamente prescriptivo. Por ello, sostienen que la comunalidad para las mujeres es más prescriptiva que lo agéntico para los hombres (Rudman \& Glick, 1999).
} 
Acorde con ello, se produciría una incongruencia, o como señala Heilman (2001), una percepción de "falta de ajuste" (lack of fit) entre los atributos estereotípicos adscritos a las mujeres y los requerimientos de cargos desempeñados tradicionalmente por hombres, especialmente cargos de dirección. Y esta percepción de incongruencia incidiría en las expectativas de desempeño sobre una persona y en la percepción y valoración de la información sobre ella en el trabajo, lo que subyace a la toma de decisiones. Entonces, una percepción de desajuste puede crear una predisposición negativa, lo que explicaría que, al momento de seleccionar a un postulante para un cargo típicamente masculino, se recomiende más a hombres que a mujeres, aunque ambos tengan idéntica preparación profesional.

Otro aspecto interesante señalado en los estudios se refiere al hecho que mujeres en cargos de dirección que muestran rasgos agénticos -más congruentes con esos cargos- pueden ser vistas como bien ajustadas a los requerimientos del puesto, y su desempeño ser evaluado como eficiente y competente. No obstante, suelen ser percibidas como no ajustadas en relación a los roles y estereotipos femeninos, pues se las ve transgrediéndolos, lo que conduce a una devaluación o penalización en tanto personas. Así, mujeres que ejercen liderazgos agénticos, asertivos y fuertes son evaluadas como menos agradables socialmente, más frías y menos hábiles en las relaciones interpersonales, en comparación con hombres agénticos (Eagly \& Carli, 2007; Eagly \& Karau, 2002; Heilman, 2001; Heilman et al., 2004; Schein, 2001). Sin embargo, es posible esperar que, bajo ciertas situaciones, esta negativa evaluación de mujeres agénticas sea moderada, cuando ellas exhiben rasgos agénticos relacionados con la competencia (autoconfianza, seguridad, individualismo, ambición) y no relacionados con dominación social (competitividad, agresividad, fortaleza, firmeza, decisión), asociados con una percepción de transgresión más directa de las normas prescriptivas de género femenino (Rudman \& Glick, 2001). En consecuencia, ya que la competencia de las mujeres para el trabajo no necesariamente entra en conflicto con la prescripción de sus rasgos comunales, se podría esperar que las sanciones hacia las mujeres agénticas competentes no dominantes socialmente sean suavizadas. De este modo, se podría dar el consistente patrón respecto de una mayor predilección del estereotipo femenino por sobre el masculino o "efecto de las mujeres son maravillosas" (women-are-wonderful effect) (Eagly \& Mladinic, 1994, p. 13), que se expresa en una percepción más positiva de las mujeres como personas.

Por ultimo, aunque hombres y mujeres tienden a expresar actitudes más positivas hacia hombres en posiciones de liderazgo (Catalyst, 2007; García-Retamero \& LópezZafra, 2006), algunas investigaciones sugieren que las mujeres en cargos de dirección perciben que tanto mujeres como hombres pueden poseer características necesarias para ejercer un rol de liderazgo. En cambio, los hombres en general, y aquellos en cargos de dirección, perciben que es más probable que los hombres posean características para un liderazgo exitoso y son quienes manifiestan más dudas respecto de la efectividad del liderazgo femenino, juzgando más duramente a las mujeres en posiciones de autoridad (Schein, 2001).

De acuerdo con lo planteado, el estudio se propuso medir la evaluación que recibía, en el ámbito laboral y en el plano personal, un hombre y una mujer con rasgos agénticos competentes, que desempeñaba un cargo gerencial ocupado tradicionalmente por hombres (cargo masculinizado); examinar los posibles sesgos que podrían surgir de los roles y estereotipos de género presentes en la evaluación que recibía cada gerente; y determinar si existían diferencias en la evaluación de acuerdo al sexo del evaluador.

Se formuló como hipótesis general que en el ámbito laboral el hombre gerente recibe una evaluación mayor a la que recibe la mujer gerente; en tanto, en el ámbito personal es la mujer gerente quien recibe una mayor evaluación.

\section{Método}

\section{Diseño}

Se realizó un diseño cuasi experimental de tipo transversal e inter sujeto. 
Las variables independientes fueron Sexo de los Gerentes Evaluados y Sexo de los Evaluadores. Se midió su efecto sobre dos variables dependientes: Evaluación en el Ámbito Laboral y Evaluación en el Ámbito Personal. En la variable Ámbito Laboral se distinguieron dos dimensiones: Efectividad del Liderazgo y Recomendaciones sobre Recompensas Organizacionales. En la primera dimensión se distinguieron tres variables que la literatura ha definido como los criterios más frecuentes en la evaluación del liderazgo efectivo (ver Tabla 1; Heilman et al., 2004; Muchinsky, 2007). La evaluación en el Ámbito Laboral consistió en el promedio de la suma de las evaluaciones en cada una de las dos dimensiones; en tanto en el Ámbito Personal se solicitó a los participantes que hicieran una evaluación como persona del/la gerente descrito/a en el escenario.

\section{Participantes}

La muestra fue intencionada, seleccionada de acuerdo a los siguientes criterios: hombres y mujeres profesionales que preferentemente ocuparan cargos en los cuales tuvieran personas bajo su responsabilidad y que participaran en procesos de evaluación y contratación de personas. Participaron 242 personas (123 hombres y 119 mujeres), con una edad promedio de 35 años $(D S=8,3)$ y 12 años promedio de experiencia laboral (DS $=8,7$ ). Todos eran profesionales, predominantemente ingenieros de distintas especialidades, y casi un 80\% desempeñaba cargos en los que tenían personas bajo su responsa- bilidad, en empresas de distintos rubros ubicadas en la ciudad de Santiago de Chile.

\section{Instrumento}

Estuvo compuesto por dos escenarios (Gormaz, 2008), uno que presentaba a un hombre con rasgos agénticos que se desempeñaba como gerente de finanzas en una entidad bancaria y otro que describía a una mujer con rasgos agénticos desempeñando el mismo cargo. Se trató de escenarios que describían a una persona eficiente, competente y exitosa, en los que solo cambiaba el nombre (masculino o femenino) de la persona. Los escenarios iban acompañados de un cuestionario para evaluar a cada gerente en el ámbito laboral (Gormaz, 2008) y un cuestionario para evaluarle en el ámbito personal. El formato de respuesta fue una escala de evaluación de siete puntos. Por ejemplo, en una primera sección los participantes evaluaron el grado de presencia o ausencia $(1=$ ausencia total, $2=$ presencia muy leve, 3 $=$ presencia leve, $4=$ presencia promedio, $5=$ presencia moderada, $6=$ presencia elevada, $7=$ presencia muy elevada) de un listado de características, tales como amabilidad, ambición, iniciativa, habilidad para trabajar en equipo, seguridad en sí mismo/a. Posteriormente, debían responder a preguntas como ¿Cuán exitosa considera que ha sido esta persona en el trabajo actual? (desde $1=$ nada exitosa hasta $7=$ muy exitosa) 0 , dentro del plano personal, ¿Cuán agradable considera a esta persona? (desde $1=N a d a$ agradable hasta $7=$ Muy agradable) .

Tabla 1

Variables Dependientes

\begin{tabular}{|l|l|l|}
\hline \multirow{2}{*}{$\begin{array}{l}\text { Ámbito } \\
\text { Laboral }\end{array}$} & Efectividad de Liderazgo & Medidas relativas a la orientación a la tarea \\
\cline { 2 - 3 } & $\begin{array}{l}\text { Medidas relativas a la orientación } \\
\text { interpersonal }\end{array}$ \\
\cline { 2 - 3 } & $\begin{array}{l}\text { Recomendaciones sobre } \\
\text { Recompensas Organizacionales }\end{array}$ & Salarios, contratación, retención y promoción \\
\hline \multirow{2}{*}{$\begin{array}{l}\text { Ámbito } \\
\text { Personal }\end{array}$} & $\begin{array}{l}\text { Evaluación general como persona fuera del ámbito laboral, nivel de agradabilidad, } \\
\text { si recurrirían a esta persona para solicitar apoyo o consejo ante un problema } \\
\text { personal, si establecerían una relación de amistad con ella. }\end{array}$ \\
\hline
\end{tabular}


El análisis de confiabilidad indicó que el instrumento muestra para cada una de las variables dependientes una alta consistencia interna (Tabla 2).

\section{Procedimiento}

Se utilizó el Paradigma de Goldberg (Eagly \& Mladinic, 1994), presentando un escenario a cada participante: para un grupo, el nombre de la persona descrita en el escenario era masculino y, para otro, femenino. Las aplicaciones del instrumento se hicieron a través de tres modalidades: aplicaciones grupales en cursos de MBA de distintas universidades y aplicaciones individuales en formato escrito y en formato on line. A todos los participantes se les invitó a participar en un estudio sobre evaluación de efectividad del liderazgo de gerentes, explicándoles que su colaboración era absolutamente voluntaria y que toda la información que entregaran sería anónima y utilizada solo para fines académicos. Cada participante recibía un escenario y dos cuestionarios, debiendo realizar primero la evaluación de la persona que aparecía en el escenario en el ámbito laboral y, posteriormente, en el ámbito personal. Las aplicaciones se realizaron desde el mes de diciembre de 2006 a septiembre de 2007. Un 48\% de los hombres participantes evaluó el escenario que presentaba a un hombre gerente y un $52 \%$, el que presentaba a una mujer gerente. Un 52\% de las mujeres evaluó el escenario que presentaba al hombre gerente y un $48 \%$, el que presentaba a la mujer gerente.

\section{Análisis de la Información}

Se realizó un análisis univariado de la varianza (ANOVA) de dos factores (sexo de evaluador y sexo del evaluado) para cada una de las variables consideradas. Adicionalmente, para analizar las diferencias existentes entre las evaluaciones que recibió cada gerente en el ámbito laboral y en el ámbito personal, se realizó un análisis multivariado de la varianza (MANOVA) 2 × 2 × 2 (sexo del evaluador, sexo del evaluado y ámbito de evaluación), siendo las dos últimas medidas repetidas, por cuanto cada participante evaluó a un gerente en dos dimensiones, ámbito laboral y ámbito personal.

\section{Resultados}

Es preciso señalar que como cada participante evaluó solo un escenario, cada vez que se indique que se encontraron (o no se encontraron) diferencias significativas en la evaluación otorgada por las mujeres a un gerente, se estará haciendo referencia a la presencia (o ausencia) de diferencias entre las evaluaciones del grupo de mujeres que evaluó a la mujer gerente y el grupo de mujeres que evaluó al hombre gerente. El mismo comentario es pertinente en el caso de los evaluadores hombres.

Tabla 2

Alfa de Cronbach del Instrumento para las Variables Dependientes Evaluación Ámbito Laboral y Evaluación Ámbito Personal

\begin{tabular}{lc}
\hline Dimensiones & Alfa de Cronbach \\
\hline Evaluación Ámbito Laboral & 0,967 \\
Efectividad de Liderazgo & 0,961 \\
Medidas de Orientación a la Tarea & 0,955 \\
Medidas de Orientación Interpersonal & 0,917 \\
Habilidades Cognitivas & 0,822 \\
Recompensas Organizacionales & 0,927 \\
Evaluación Ámbito Personal & 0,913 \\
\hline
\end{tabular}




\section{Evaluación en el Ámbito Laboral}

En relación a la evaluación en el ámbito laboral, formada por el promedio de la suma de las evaluaciones de Efectividad de Liderazgo y las Recomendaciones de Recompensas Organizacionales, se hipotetizó que no se observarían diferencias significativas en la evaluación que las mujeres realizaran del hombre gerente y de la mujer gerente; en cambio, que los hombres otorgarían una evaluación significativamente mayor al hombre gerente.

Contrario a lo esperado, no se observaron diferencias entre la evaluación que recibió el hombre gerente y la mujer gerente, pues, con independencia del sexo de los evaluadores, fueron evaluados de manera similar: sexo de los gerentes $F(1,241)=0,728$, $p=0,394$; sexo de los evaluadores $F(1,241$ $=0,568, p=0,452$; sexo de los evaluadores y sexo de los gerentes $F(1,241)=2,438, p$ $=0,120$. Los promedios de las evaluaciones otorgadas pueden verse en la Tabla 3 .

Las respuestas en cada uno de los niveles de esta variable muestra el mismo patrón. No se observaron diferencias en la evaluación que recibió cada gerente en relación a la Efectividad de Liderazgo, formada por el promedio de la suma de las evaluaciones de las dimensiones Medidas de Orientación a la Tarea, Medidas de Orientación Interpersonal y Habilidades Cognitivas: sexo de los gerentes $F(1,241)=0,739, p=0,391$; sexo de los evaluadores $F(1,241)=0,335, p$ $=0,564 ;$ sexo de los evaluadores y sexo de los gerentes $F(1,241)=2,510, p=0,114$.

Tampoco se observaron diferencias en relación a las Recomendaciones sobre Recompensas Organizacionales: sexo del gerente $F(1,241)=0,469, p=0,494$; sexo de los evaluadores $F(1,241)=0,843, p=0,359$; sexo de los evaluadores y sexo de los gerentes $F(1,241)=1,52, p=0,219$.

Similares resultados se observaron en dos de las variables a través de las cuales se evaluó la Efectividad de Liderazgo (Orientación a la Tarea y Habilidades Cognitivas), en tanto en la evaluación de las Medidas de Orientación Interpersonal se observó un efecto de interacción significativo entre sexo del evaluador y sexo del gerente, $F(1,241)=$ $4,490, p=0,035$, que, de acuerdo al análisis de efectos simples, las mujeres otorgaron una evaluación mayor a la mujer gerente, $F(1,241)=4,08, p=0,045, \mathrm{y}$ los hombres otorgaron una mayor evaluación al hombre gerente, $F(1,241)=7,73, p=0,006$.

Tabla 3

Evaluaciones Promedio del Hombre Gerente y la Mujer Gerente en el Ámbito Laboral en General y en la Efectividad del Liderazgo y las Recompensas Organizacionales, según Sexo del Evaluador

\begin{tabular}{|c|c|c|c|c|c|c|}
\hline \multirow{2}{*}{ Ámbito y Dimensiones } & \multicolumn{2}{|c|}{ Evaluador Hombre } & \multicolumn{2}{|c|}{ Evaluadora Mujer } & \multicolumn{2}{|c|}{ Total } \\
\hline & $M$ & $D S$ & $M$ & $D S$ & $M$ & $D S$ \\
\hline \multicolumn{7}{|l|}{ Ámbito Laboral } \\
\hline Hombre Gerente & 5,74 & 0,694 & 5,50 & 1,126 & 5,61 & 0,944 \\
\hline Mujer Gerente & 5,66 & 0,579 & 5,75 & 0,661 & 5,70 & 0,618 \\
\hline Total & 5,70 & 0,635 & 5,62 & 0,937 & 5,66 & 0,797 \\
\hline \multicolumn{7}{|l|}{ Efectividad del Liderazgo } \\
\hline Hombre Gerente & 5,72 & 0,634 & 5,52 & 1,050 & 5,62 & 0,875 \\
\hline Mujer Gerente & 5,65 & 0,513 & 5,75 & 0,545 & 5,70 & 0,529 \\
\hline $\begin{array}{c}\text { Total } \\
\text { Recompensas }\end{array}$ & 5,69 & 0,573 & 5,63 & 0,851 & 5,66 & 0,722 \\
\hline \multicolumn{7}{|l|}{$\begin{array}{l}\text { Recompensas } \\
\text { Organizacionales }\end{array}$} \\
\hline Hombre Gerente & 5,76 & 1,059 & 5,42 & 1,589 & 5,59 & 1,362 \\
\hline Mujer Gerente & 5,67 & 1,023 & 5,73 & 1,244 & 5,70 & 1,128 \\
\hline Total & 5,72 & 1,037 & 5,56 & 1,437 & 5,64 & 1,249 \\
\hline
\end{tabular}




\section{Evaluación en el Ámbito Personal}

Se hipotetizó que no se observarían diferencias significativas en la evaluación que hombres y mujeres realizaran de la mujer gerente, en tanto la evaluación del hombre gerente sí mostraría diferencias significativas, recibiendo una mayor evaluación de parte de los hombres.

Contrario a lo esperado, no se observaron diferencias significativas en la evaluación que otorgaron hombres y mujeres al hombre gerente ni tampoco en la evaluación que otorgaron a la mujer gerente: sexo de los evaluadores $F(1,240)=2,620, p=$ 0,107 ; sexo de los gerentes $F(1,240)=0,419$, $p=0,518$; sexo de los evaluadores y sexo de los gerentes $F(1,240)=0,703, p=0,403$. Los promedios de las evaluaciones otorgadas pueden verse en la Tabla 4.

Comparación entre la Evaluación en el Ámbito Laboral y el Ámbito Personal

Se hipotetizó que no se observarían diferencias significativas en la evaluación $l a$ boral y personal que otorgaran los hombres al gerente hombre ni en la evaluación que otorgaran las mujeres a la mujer gerente; en tanto, sí se observarían diferencias significativas en la evaluación laboral y personal de los hombres a la mujer gerente (sería mayor la evaluación en el ámbito personal) y de las mujeres al hombre gerente (sería mayor la evaluación en el ámbito laboral).

Contrariamente a lo esperado, como se puede apreciar en las Tablas 3 y 4, no se produjeron diferencias significativas respecto del sexo del gerente o del sexo de los evaluadores. Las diferencias se encontraron en el ámbito en el que se realizaba la evaluación. El análisis de medidas repetidas mostró que las evaluaciones que recibió cada gerente en el ámbito laboral son mayores a las que recibió en el ámbito personal, $F(1,237)=200,97, p<0,000)$.

Estos resultados no confirman la hipótesis general, pues en el ámbito laboral el hombre gerente no recibió una evaluación mayor en comparación con la que recibió la mujer gerente; y en el ámbito personal, la mujer gerente no recibió una mayor evaluación a la del hombre gerente. En ambos ámbitos no se observaron diferencias significativas en la evaluación de los gerentes. Sin embargo, las evaluaciones recibidas por un mismo gerente en el ámbito laboral y el ámbito personal sí mostraron diferencias significativas, pues hombres y mujeres otorgaron a ambos gerentes evaluaciones significativamente mayores en el ámbito laboral.

\section{Discusión}

Uno de los hallazgos que más llama la atención es la ausencia de diferencias significativas en la evaluación que recibió el hombre gerente y la mujer gerente en el ámbito laboral, y más aún, que ello ocurra con independencia del sexo de los evaluadores. Esta similitud de las evaluaciones se dio tanto en relación a sus habilidades para tener éxito en las tareas encomendadas y lograr los objetivos (Efectividad de Liderazgo) como en las recomendaciones de promoción, contratación y aumentos salariales que reciben (Recompensas Organizacionales). En

Tabla 4

Evaluaciones Promedio del Hombre Gerente y la Mujer Gerente en el Ámbito Personal, según Sexo del Evaluador

\begin{tabular}{lcccccc}
\hline \multirow{2}{*}{ Sexo del Gerente } & \multicolumn{2}{c}{ Evaluador Hombre } & \multicolumn{2}{c}{ Evaluadora Mujer } & \multicolumn{3}{c}{ Total } \\
\cline { 2 - 7 } & $M$ & $D S$ & $M$ & $D S$ & $M$ & $D S$ \\
\hline Hombre Gerente & 4,64 & 1,40 & 4,26 & 1,71 & 4,45 & 1,57 \\
Mujer Gerente & 4,48 & 1,38 & 4,36 & 1,44 & 4,42 & 1,41 \\
Total & 4,56 & 1,39 & 4,31 & 1,58 & 4,44 & 1,49 \\
\hline
\end{tabular}


el ámbito personal, en el que se esperaba que la mujer gerente recibiera una evaluación mayor a la otorgada al hombre gerente, nuevamente no se observan diferencias significativas. No obstante, se advierte que las evaluaciones que ambos gerentes obtienen en el ámbito personal son significativamente inferiores a las que reciben en el ámbito laboral.

Esta ausencia de diferencias significativas en la evaluación que recibe el hombre gerente y la mujer gerente en el ámbito laboral ¿expresa el fin de los prejuicios y resistencias que tradicionalmente han dificultado a las mujeres el acceso a cargos de dirección? Y el hecho que la evaluación de ambos gerentes en el ámbito personal sea inferior a la que reciben en el ámbito laboral ¿significa que las competencias agénticas afectan negativamente no solo la evaluación de las mujeres en el plano personal, sino también la de los hombres?

Más que dar una respuesta definitiva a estas preguntas, los resultados obtenidos permiten sostener que, bajo determinadas circunstancias, estos prejuicios pueden disminuir significativamente.

La ausencia de diferencias significativas en el ámbito laboral parece confirmar lo encontrado en anteriores investigaciones respecto que la percepción más estereotípica de mujeres comunales y hombres agénticos se debilita cuando se trata de mujeres managers exitosas. Como lo indican Heilman, Block y Martell (1995), se puede producir una caracterización de las mujeres que ocupan cargos de dirección -sobre todo cuando son exitosas- como personas con rasgos más agénticos, en comparación con la percepción de las mujeres en general.

Estos resultados se relacionan también con el hecho que el rol ocupacional tendría un peso mayor que el rol de género al momento de evaluar a personas que se desempeñan en cargos directivos (Eagly \& Mladinic, 1994). Los evaluadores tendrían en cuenta el rol ocupacional de la mujer y el hombre evaluado, más que sus roles de género, al momento de evaluarlos en relación a la efectividad de liderazgo y al hacer recomendaciones de recompensas organizacionales.

Los resultados también parecen refle- jar la tendencia más general respecto que las mujeres estarían viéndose a sí mismas, y siendo vistas por otros, como más agénticas (Eagly \& Carli, 2004; Rudman \& Glick, 1999, 2001), a lo que la mayor participación laboral femenina ha contribuido de manera relevante. Esta visión sobre las mujeres expresaría una flexibilización de los roles y estereotipos de género fundamentalmente por la incorporación al estereotipo femenino de muchos rasgos considerados tradicionalmente masculinos, sin eliminar otros considerados tradicionalmente femeninos (Baron \& Byrne, 2004; Eagly \& Mladinic, 1994). En este sentido, Rudman y Glick (1999, 2001) afirman que la visión más agéntica de las mujeres no ha llevado a que ellas dejen de ser categorizadas como personas con rasgos comunales. Es decir, como lo indican otras investigaciones, los cambios en los roles de género que permiten adaptarse a nuevas realidades sociales se instalan en convivencia con modelos tradicionales que permanecen (Sharim, 2005). De acuerdo con lo señalado, el hecho que evaluadores hombres y mujeres consideren que ambos gerentes muestran similares niveles de capacidad para desempeñar un cargo tradicionalmente ocupado por hombres, sería una manifestación de este cambio en la forma como están siendo percibidas las mujeres y, más especialmente, aquellas insertas en el mercado laboral que se desempeñan exitosamente en cargos directivos.

De acuerdo con lo señalado, se advierten más cambios en la percepción de roles y estereotipos asociados con mujeres en cargos de dirección -tanto de parte de hombres como de mujeres- que en la percepción de los atributos necesarios para desempeñar estos cargos; es decir, los rasgos agénticos no dejan de asociarse con posiciones de liderazgo, sino que dejan de ser vistos como exclusivos de los hombres.

Respecto de la evaluación en el ámbito personal, la ausencia de diferencias en las evaluaciones que reciben los gerentes no permite confirmar la hipótesis que la mujer gerente es mejor evaluada, siguiendo así la consistente percepción más positiva de las mujeres como personas (Eagly \& Mladinic, 1994). No obstante, el hecho que esta evaluación sea significativamente inferior a la 
que reciben gerentes de ambos sexos en el ámbito laboral, indicaría que no es solo la mujer gerente con rasgos agénticos la que puede verse afectada en su evaluación en términos personales (Heilman, 2001; Heilman et al., 1995, 2004). A pesar de ello, se debe considerar que, en la medida que la comunalidad para las mujeres es más prescriptiva que lo agéntico para los hombres (Rudman \& Glick, 1999) -conductas y rasgos relacionados con el bienestar de otros son altamente deseables y admirados en las mujeres-, una evaluación que no supere el nivel promedio de características como agradabilidad o amabilidad puede tener un efecto más negativo en las mujeres que en los hombres, de quienes no necesariamente se espera este tipo de rasgos. Así, mientras en los hombres una evaluación más baja de estas características puede ser vista como una ausencia, entre las mujeres puede ser percibida como una carencia. Como lo indica Schein (2001), mientras un hombre competente tiende a ser visto como "no comunal" (noncommu$n a l)$, una mujer de iguales características ejerciendo un rol masculino es vista como "contra comunal" (countercommunal).

Por otra parte, los resultados obtenidos deben tener en cuenta algunas consideraciones metodológicas. En relación con el instrumento, los escenarios utilizados presentaban información clara, explícita y específica acerca de las cualificaciones y el desempeño laboral de los gerentes. Ello contribuyó a reducir la tendencia a que las mujeres reciban evaluaciones sesgadas por género en tareas tipificadas como masculinas, ya que, como lo indican Heilman et al. (1995, 2004), este tipo de información reduce las inferencias o interpretaciones de los evaluadores que favorecen la aparición de sesgos de género en la evaluación de mujeres en este tipo de tareas.

Con respecto a las características de la muestra, se trata de profesionales pertenecientes a generaciones jóvenes y en cargos de dirección, entre quienes existiría una percepción más positiva del trabajo remunerado femenino (Grupo Iniciativa Mujeres, 1999; Lehmann, 2003) y también, de acuerdo a los resultados, un debilitamiento de las actitudes más negativas que especialmente los hombres han expresado hacia el lide- razgo femenino. Hay que tener en cuenta, además, que se trata de hombres y mujeres que ingresaron a un mercado laboral que en las últimas décadas ha experimentado importantes transformaciones: presencia más numerosa de mujeres, surgimiento de discursos empresariales que valorizan los liderazgos femeninos, incremento de una positiva percepción del empleo femenino y una mayor visibilización y sanción social de las discriminaciones por razones de género.

Relacionado con ello, también se debe considerar el momento en el cual fue realizada la investigación, marcado por una alta recurrencia en los medios de comunicación del liderazgo femenino, en gran medida a raíz de la primera mujer Presidenta de la República. Todo esto constituye un contexto que propicia una opinión más favorable hacia las demandas de las mujeres, hacia el empleo femenino y una mayor sanción social hacia la discriminación por razones de género. Este favorable contexto incide en lo que se considera "políticamente aceptable o correcto", por lo que no se podría descartar que, a pesar del diseño utilizado para la investigación, las respuestas de los participantes expresen también deseabilidad social, es decir, un discurso público que se muestra mucho más cuidadoso al momento de hablar de las capacidades y atributos de las mujeres, especialmente en el ámbito laboral, para evitar juicios que pudieran ser calificados como discriminatorios.

\section{Conclusiones}

Los resultados obtenidos, más que expresar el fin de los prejuicios y resistencias que han dificultado el acceso de mujeres a cargos de dirección, muestran que la percepción de incongruencia esperada entre el rol de liderazgo y los roles de género femenino planteada por Eagly (1987), o la falta de ajuste entre atributos estereotípicamente femeninos y los requerimientos de cargos de dirección desempeñados tradicionalmente por hombres, planteada por Heilman (2001), se debilita bajo ciertas condiciones. Esto sucedería en circunstancias en las cuales los evaluadores cuentan con información específica, directa y precisa para hacer sus evaluaciones, cuando se trata de evaluar a 
mujeres claramente competentes y exitosas en cargos directivos, y cuando esa evaluación proviene de ejecutivos de generaciones jóvenes. En estas circunstancias, la discrepancia entre las características atribuidas a las mujeres y las asociadas a cargos de dirección disminuye a un punto tal que desaparecen las diferencias en las evaluaciones recibidas por mujeres y hombres gerentes.

Lo señalado permite extraer varias conclusiones. En relación a los procesos de evaluación, la utilización de instrumentos que contengan información explícita y precisa y un número amplio de preguntas y de formas de preguntar, que posibiliten indagar en profundidad diversos aspectos relevantes, es una condición que contribuye a disminuir los sesgos de género en dichos procesos y a un mejor aprovechamiento de las competencias profesionales de las personas, independiente de su género.

En relación al marco conceptual, el hecho de no haber confirmado una percepción de incongruencia entre roles femeninos y roles de liderazgo, más que manifestar un cambio social general, refleja los cambios en un grupo social particular: jóvenes profesionales en cargos de dirección. En este sentido, es preciso ver los alcances de este marco conceptual en condiciones muy diferentes a las de este estudio, por ejemplo, incluir entre los participantes a personas de distintos niveles jerárquicos (inferiores y superiores) y de distintas generaciones para ver si sus evaluaciones a hombres y mujeres en posiciones de dirección presentan diferencias significativas. Igualmente, resultaría relevante recoger información adicional de los participantes (más allá de su nivel de formación, cargo y experiencia laboral), que eventualmente pudiera incidir en su percepción de mujeres en cargos directivos, por ejemplo, tipo de organización, experiencias previas con jefaturas femeninas y masculinas y tipo de arreglo familiar.

Por último, la evaluación inferior que gerentes hombres y mujeres reciben en el ámbito personal es un aspecto especialmente importante de considerar en posteriores investigaciones, para explicar, no solo las dificultades que enfrentan las mujeres para acceder a cargos directivos sino, también, las que enfrentan aquellas que han llegado a estos cargos. Así, se podría indagar la forma en que mujeres ejecutivas perciben la penalización interpersonal y de qué modo ello puede dificultar su desempeño laboral, las relaciones con sus pares, superiores y subordinados y el avance en sus carreras.

\section{Referencias}

Abramo, L. \& Valenzuela, M. E. (2006). Inserción laboral y brechas de equidad de género en América Latina. En L. Abramo (Ed.), Trabajo decente y equidad de género en América Latina (pp. 9-62). Santiago, Chile: Organización Internacional del Trabajo.

Baron, R. \& Byrne, D. (2004). Psicología social (8 ${ }^{\mathrm{a}}$ ed.). Madrid: Pearson Prentice Hall.

Bass, B. M. \& Avolio, B. J.(1994). Improvingorganizational effectiveness through transformational leadership. Thousand Oaks, CA: Sage Publications.

Bass, B. M., Avolio, B. J., Jung, D. I. \& Berson, Y. (2003). Predicting unit performance by assessing transformational and transactional leadership. Journal of Applied Psychology, 88, 207-218.

Catalyst (2007). The double-bind dilemma for women in leaderships: Damned if you do, doomed if you don't. New York: Autor. Extraído el 20 Marzo, 2009, de http://www.catalyst.org/file/45/ the $\% 20$ double-bind $\% 20$ dilemma $\% 20$ for $\% 20$ wom en\%20in\%20leadership\%20damned\%20if\%20you $\% 20$ do,\%20doomed\%20if\%20you\%20don't.pdf

Centro de Estudios Empresariales de la Mujer (2008). Creación del perfil de las directoras de las sociedades anónimas cerradas y abiertas (Informe $\mathrm{N}^{\circ}$ 4). Santiago, Chile: Universidad del Desarrollo, Facultad de Economía y Negocios.

Corporación Humanas (2007). Democracia en el país, democracia en la casa, democracia paritaria. Informe regional: percepciones de las mujeres sobre su situación y condiciones de vida en Argentina, Bolivia, Chile, Ecuador y Perú. Santiago, Chile: Autor, Centro Regional de Derechos Humanos y Justicia de Género.

Chile, Ministerio de Planificación (2006). Encuesta de Caracterización Socioeconómica Nacional 2006. Trabajo e ingresos. Santiago, Chile: Autor.

Díaz, X., Godoy, L. \& Stecher, A. (2005). Significados del trabajo y vínculos sociales: dimensiones subjetivas de las transformaciones del mercado laboral (Cuaderno de Investigación $\mathrm{N}^{\circ}$ 3). Santiago, Chile: Ediciones CEM.

Eagly, A. H. (1987). Sex differences in social behavior: A social-role interpretation. Hillsdale, NJ: Lawrence Erlbaum Associates.

Eagly, A. H. \& Carli, L. L. (2004). Women and men as leaders. En J. Antonakis, A. T Cianciolo \& R. J. Sternberg (Eds.), The nature of leaderships (pp. 279-301). Thousand Oaks, CA: Sage Publications. 
Eagly, A. H. \& Carli, L. L. (2007). Women and the labyrinth of leadership. Harvard Business Review, September, 62-71.

Eagly, A. H. \& Karau, S. J. (1991). Gender and the emergence of leaders: A meta-analysis. Journal of Personality \& Social Psychology, 60, 685-710.

Eagly, A. H. \& Karau, S. J. (2002). Role congruity theory of prejudice toward female leaders. Psychological Review, 109, 573-598.

Eagly, A. H., Karau S. J. \& Makhijani, M. G. (1995). Gender and the effectiveness of leaders: A metaanalysis. Psychological Bulletin, 117, 125-145.

Eagly, A. H., Makhijani, M. G. \& Klonsky, B. G. (1992). Gender and the evaluation of leaders: A metaanalysis. Psychological Bulletin, 111, 3-22.

Eagly, A. H. \& Mladinic, A. (1994). Are people prejudiced against women? Some answers from research on attitudes, gender stereotypes, and judgments of competence. European Review of Social Psychology, 5, 1-35.

Eagly, A. H., Wood, W. \& Johannesen-Schmidt, M. C. (2004). Social roles theory of sex differences and similarities: Implications for the partner preferences of women and men. En A. H. Eagly, A. Beall \& R. J. Sternberg (Eds.), The psychology of gender ( $2^{\text {nd }}$ ed., pp. 269-295). New York: Guilford Press.

García-Retamero, R. \& López-Zafra, E. (2006). Prejudice against women in male-congenial environments: Perceptions of gender role congruity in leadership. Sex Roles, 55, 51-61.

Godoy, L., Stecher, A. \& Díaz, X. (2007). Trabajo e identidades: continuidades y rupturas en un contexto de flexibilización laboral. En R. Guadarrama \& J. L. Torres (Coords.), Los significados del trabajo femenino en el mundo global: estereotipos, transacciones y rupturas (pp. 81-100). Barcelona: Anthropos.

Gormaz, N. (2008). Diferencias de género en el liderazgo organizacional: ¿ Por qué tan pocas mujeres llegan a la cima? Tesis no publicada para optar al grado de Doctora en Psicología, Pontificia Universidad Católica de Chile, Santiago, Chile.

Grupo Iniciativa Mujeres (1999). Encuesta nacional. Opinión y actitudes de las mujeres chilenas sobre la condición de género. Santiago, Chile: Autor.

Guzmán, V., Mauro, A. \& Araujo, K. (1999). Trayectorias laborales de mujeres. Cambios generacionales en el mercado de trabajo. Santiago, Chile: Ediciones CEM.

Heilman, M. E. (2001). Description and prescription: How gender stereotypes prevent women's ascent up the organizational ladder. Journal of Social Issues, 57, 657-673.

Heilman, M. E., Block, C. J. \& Martell, R. F. (1995). Sex stereotypes: Do they influence perceptions of managers? Journal of Social Behavior and Personality, 10, 237-252.

Heilman, M. E., Wallen, A. S., Fuchs, D. \& Tamkins, M. M. (2004). Penalties for success: Reactions to women who succeed at male gender-typed tasks. Journal of Applied Psychology, 89, 416-427.

Hola, E. \& Todaro, R. (1992). Los mecanismos del poder. Hombres y mujeres en la empresa moderna. Buenos Aires: Grupo Editorial Latinoamericano.
Lehmann, C. (2003). Mujer, trabajo y familia: realidad, percepciones y desafíos. Análisis sobre la base de la Encuesta CEP de diciembre de 2002 (Puntos de Referencia $\mathrm{N}^{\mathrm{o}}$ 269). Santiago, Chile: Centro de Estudios Públicos.

Maruani, M. (1993). La cualificación, una construcción social sexuada. Economía y Sociología del Trabajo, 21-22, 41-50.

Maxfield, S. (2005). Mujeres en el límite. Poder corporativo en América Latina. Boston: Women's Leadership Conference of the Americas/InterAmerican Dialogue/Simmons Graduate School Of Management. Extraído el 3 Agosto, 2008, de http://www.simmons.edu/som/docs/centers/Al_ limite._Edicion_final.pdf

Muchinsky, P. M. (2007). Psicología aplicada al trabajo ( $8^{a}$ ed.). Ciudad de México: Thomson.

Myers, D. G. (2005). Psicología social (8 ${ }^{\mathrm{a}}$ ed.). Ciudad de México: McGraw Hill/Interamericana de México.

Organización Internacional del Trabajo (1997). El acceso de las mujeres a puestos de dirección. Genève: Autor.

Organización Internacional del Trabajo (2007). Tendencias mundiales del empleo de las mujeres. Resumen marzo de 2007. Genève: Autor. Extraído el 21 Marzo, 2009, de http://www.ilo.org/public/ spanish/employment/strat/download/getw07.pdf

Reygadas, L. (1998). Fábricas con aroma de mujer. Las nuevas culturas del trabajo vistas desde la óptica de género. En L. Abramo \& A. Rangel de Paiva Abreu (Orgs.), Gênero e trabalho na sociologia latinoamericana (pp. 101-115). São Paulo: Asociación Latinoamericana de Sociología del Trabajo/Secretaria do Emprego e Relações do Trabalho do Estado de São Paulo.

Rivera, D. \& Guajardo, G. (1996). Transformaciones en la familia con motivo de la incorporación de la mujer en el trabajo (Documento de Trabajo $\mathrm{N}^{\circ} 49$ ). Santiago, Chile: Servicio Nacional de la Mujer.

Rudman, L. A. \& Glick, P. (1999). Feminized management and backlash toward agentic women: The hidden costs to women of a kinder, gentler image of middle managers. Journal of Personality and Social Psychology, 77, 1004-1010.

Rudman, L. A. \& Glick, P. (2001). Prescriptive gender stereotypes and backlash toward agentic women. Journal of Social Issues, 57, 743-762.

Sarrió, M., Ramos, A. \& Candela, C. (2004). Género, trabajo y poder. En E. Barberá, I. Martínez \& A. Bonilla (Coord.), Psicología y género (pp. 193216). Madrid: Pearson Educación.

Schein, V. E. (2001). A global look at psychological barriers to women's progress in management. Journal of Social Issues, 57, 675-688.

Servicio Nacional de la Mujer (2001). Relaciones de género y proyectos de vida en la infancia y adolescencia chilenas. Proposiciones, 32, 242259.

Servicio Nacional de la Mujer (2005). Directivas de organizaciones empresariales (Estadísticas: Participación). Santiago, Chile: Autor. Extraído el 20 Marzo, 2009, de http://www.sernam.cl/cedocvi/basemujer/docs/CON(3)_20080704_162627.xls 
Sharim, D. (2005). La identidad de género en tiempos de cambio: una aproximación desde los relatos de vida. Psykhe, 14(2), 19-32.

Sharim, D. \& Silva, U. (1998). Familia y reparto de responsabilidades (Documento de Trabajo $\mathrm{N}^{\circ}$ 58). Santiago, Chile: Servicio Nacional de la Mujer.

Valenzuela, M. E. \& Reinecke, G. (Eds.) (2000). ¿Más y mejores empleos para las mujeres? La experiencia de los países en el MERCOSUR y en Chile.

Fecha de recepción: Noviembre de 2008.

Fecha de aceptación: Septiembre de 2009.
Santiago, Chile: Organización Internacional del Trabajo.

Yáñez, S. \& Godoy, L. (2008). Effects of gender images and stereotypes on female E\&T research careers in higher education in Chile. En A. S. Godfroy-Genin (Ed.), Women in engineering and technology research. Proceedings of the PROMETEA Conference. 26-27 Octubre 2007, Paris. Zürich: Lit Verlag. 